\title{
Correlative Fluorescence and Electron Spectroscopic Imaging of Nuclear Bodies and Domains
}

\author{
David P. Bazett-Jones, Christopher H. Eskiw, Graham Dellaire, Rosa Nisman \\ Program in Cell Biology, The Hospital for Sick Children, 555 University Avenue, Toronto, ON \\ Canada M5G 1 X8
}

The mammalian eukaryotic nucleus is a highly structured organelle containing a variety of specialized domains that accumulate proteins of specific functions. Promyelocytic leukemia (PML) bodies are an example of a sub-nuclear domain. PML protein, a major component of these bodies, has been implicated in many cellular pathways, including control of apoptosis, viral pathogenicity, and control of higher order chromatin structure [1]. Also known as nuclear domain 10 (ND10), Kremer bodies (Kr), and PML oncogeneic domains (PODs), PML bodies range in size from $250 \mathrm{~nm}$ to $1 \mu \mathrm{m}$ in diameter, and number from approximately 5 to 30 , depending on the cell type (reviewed in [2].

We have used heat shock stress as a tool to study the principles underlying the assembly, integrity, size, location and dynamic behaviour of PML bodies. Environmental stresses such as heat shock or chemical shock cause the dissociation of PML bodies into many smaller punctate domains, which we refer to as microstructures. Through a combination of live-cell imaging, immunofluorescence microscopy and energy filtered transmission electron microscopy or electron spectroscopic imaging (ESI), we observe that PML bodies are positionally stable over time intervals of a few hours. They are tightly constrained by blocks of chromatin and can only oscillate or "jiggle" with an irregular amplitude and frequency within these domains in an energy-dependent manner. Observations with ESI indicate that the bodies are associated with nascent transcripts and accumulation of RNPs. After stress, however, PML bodies are no longer sites of transcript accumulation, and have a looser association with chromatin. The microstructures arise directly from the "parental" PML bodies.

Fluorescence microscopy and ESI indicate that microstructures show no permanent engagement with chromatin and energy depletion does not affect microstructure diffusion rates. All microstrucutres eventually fuse with parental PML body remnants during recovery from heat stress. Since new PML bodies do not form at new locations, and the relative sizes observed before heat shock are preserved after recovery, we conclude that there are pre-determined locations for PML bodies, their formation is highly regulated, and that they are not random accumulations of protein. Light treatment of cells with DNase I to digest chromatin around PML bodies, followed by ESI of the treated cells, indicates that PML bodies are positionally stable due to contacts with chromatin domains.

References:

[1] G.G. Maul, D. Negorev, P. Bell, and A.M. Ishov . J. Struct. Biol. 129 (2000), 278.

[2] C.H. Eskiw and D.P. Bazett-Jones Biochem. Cell Biol. 80 (2002), 301. 

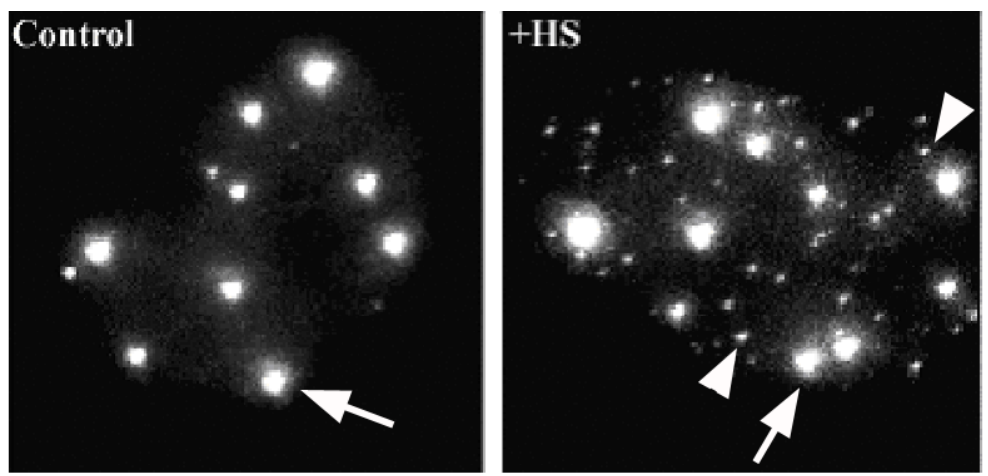

Figure 1 Immunofluorescence microscopy of PML-containing "microstructures" (arrowheads) form when cells are exposed to stress such as heat shock. They arise from the parental PML bodies (arrows). Full field represents 15 microns.
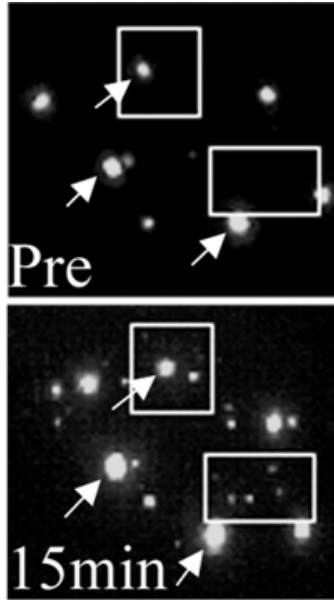
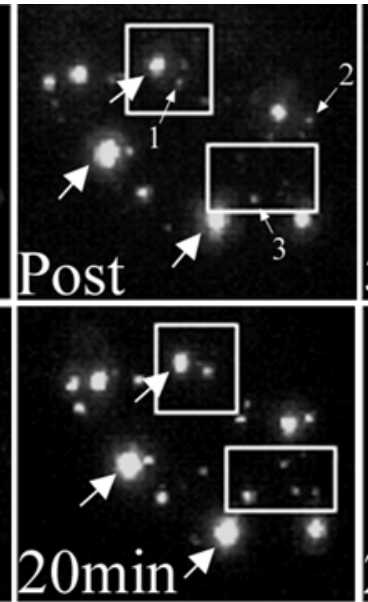
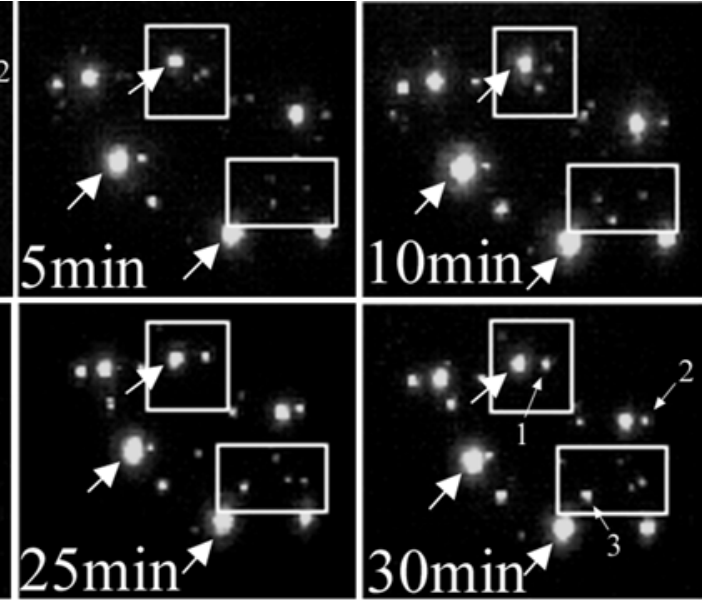

Figure 2 PML- containing microstructures are mobile as indicated from GFP-PML fluorescence time-lapse imaging. The microstructures move throughout the nucleoplasm and fuse with each, becoming larger over time (see objects labelled 1-3). Pre and post represent images recorded before and immediately following heat stress, followed by time of recovery from stress.
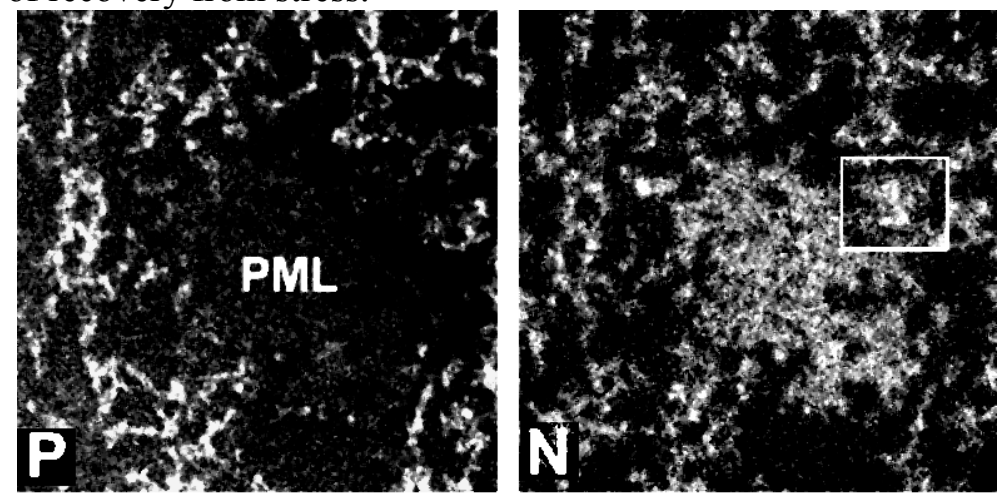

Figure 3 Dynamic behaviour of PML bodies and microstructures can be understood when examined by correlative fluorescence microscopy and ESI. Net P and net $\mathrm{N}$ images are shown here. The core of the PML body domain is P-poor, indicating it contains no detectable nucleic acid, where the core is $\mathrm{N}$-rich, indicating it is protein-based. A microstructure, identified through correlative fluorescence imaging is indicated by the box. The microstructure has moved through the nucleoplasm until abutting against a parental PML body remnant during recovery from heat stress. Full field corresponds to $750 \mathrm{~nm}$. 